\title{
Urea-Positive Providencia stuartii
}

National Cancer Institute

\section{Source}

National Cancer Institute. Urea-Positive Providencia stuartii. NCI Thesaurus. Code

C124364.

A subtype of Providencia stuartii that are positive for urease enzyme activity and are capable of hydrolyzing urea to ammonia and carbon dioxide. The determinant for urease likely resides on a transmissible plasmid. 